\title{
Dynamics of a Leslie-Gower type predation model with a non-monotonic functional response
}

\author{
EDUARDO GONZALEZ-OLIVARES ${ }^{1}$, Paulo Tintinago-Ruiz ${ }^{2}$, Lina Gallego-Berrío ${ }^{2}$, and \\ Leonardo Restrepo-Alape ${ }^{3}$ \\ ${ }^{1}$ Pontificia Universidad Catolica de Valparaiso \\ ${ }^{2}$ Universidad del Quindío \\ ${ }^{3}$ Universidad del Tolima
}

May 5, 2020

\begin{abstract}
In the ecological literature, many models for the predator-prey interactions consider the monotonic functional responses to describe the action of the predators. However, there exist antipredator behaviors which are best represented by non-monotonic functions. The mathematical results on the predator-prey models provide very useful information to understand the complex food webs; they also help to the insight of the mechanisms that govern the evolution of ecological systems. The aim of this paper is to show, the dynamics of a modified Leslie-Gower model, assuming a rational non-monotonic functional response or Holling type IV. A principal target is to compare the obtained properties with other cases, in which different non-monotonic functional responses are incorporated. The model is described by an autonomous bi-dimensional ordinary differential equation system (ODEs), assuming that the prey and predator growth functions are the logistic type. The proposed model is not defined in $\$(0,0) \$$; considering a topological equivalent system, it is possible that to prove the origin is a non-hyperbolic saddle point. We also have established, there are subsets of the parameter space in which: i) there exists a unique positive equilibrium point, ii) a heteroclinic curve exists. iii) two concentric limit cycles exist, the innermost unstable and the outermost stable. Numerical simulations are given to endorse the analytical results and to exhibit the richness of the dynamics in the system.
\end{abstract}

\section{Hosted file}

NONMONLESLIEIwileyNJD-AMA .pdf available at https ://authorea.com/users/303582/articles/433776dynamics-of-a-leslie-gower-type-predation-model-with-a-non-monotonic-functional-response 


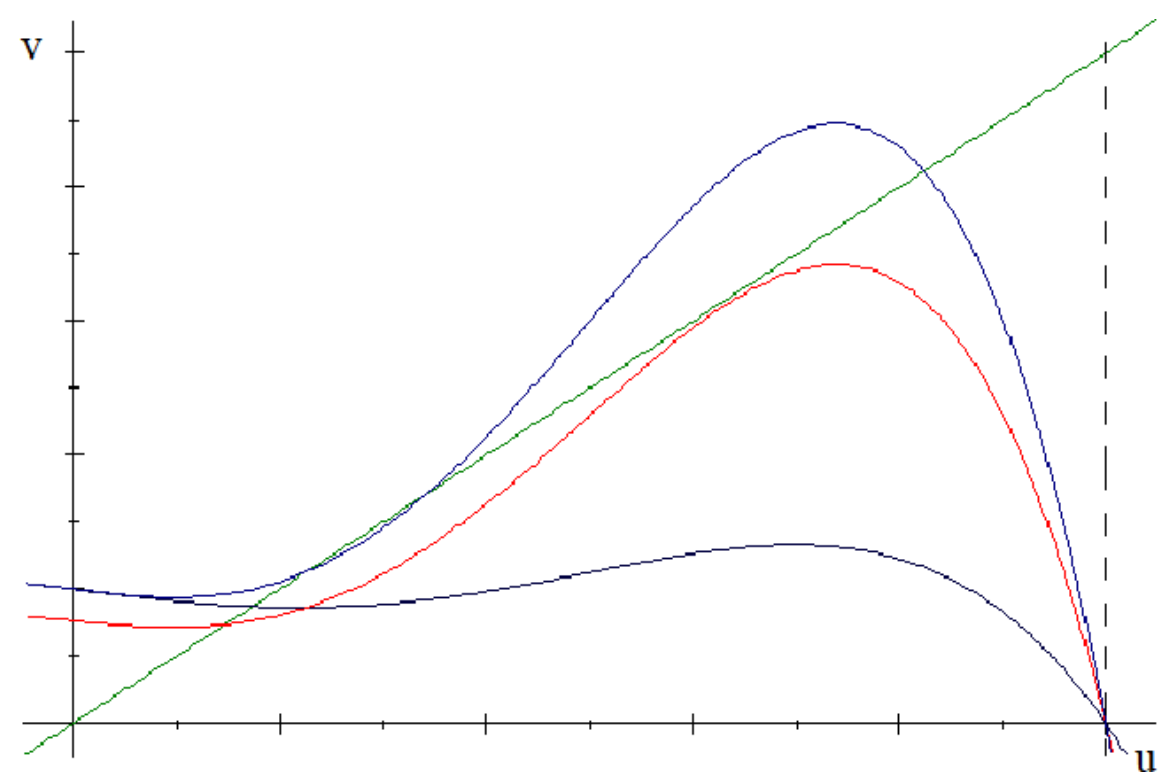

figures/fa1/fa1-eps-converted-to.pdf 
figures/fr2/fr2-eps-converted-to.pdf 
figures/cl2/cl2-eps-converted-to.pdf 
figures/sim1/sim1-eps-converted-to.pdf

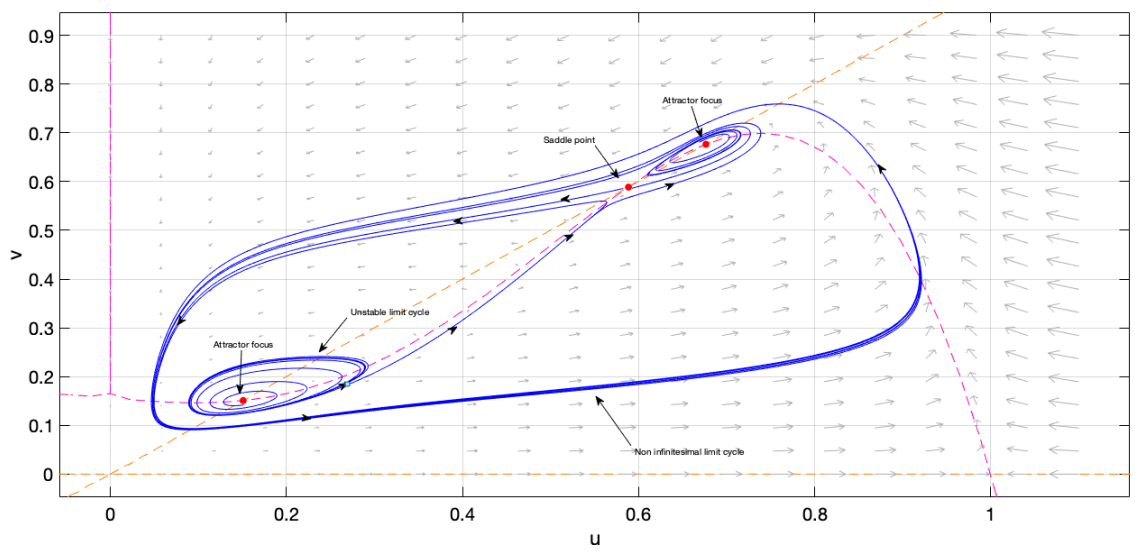



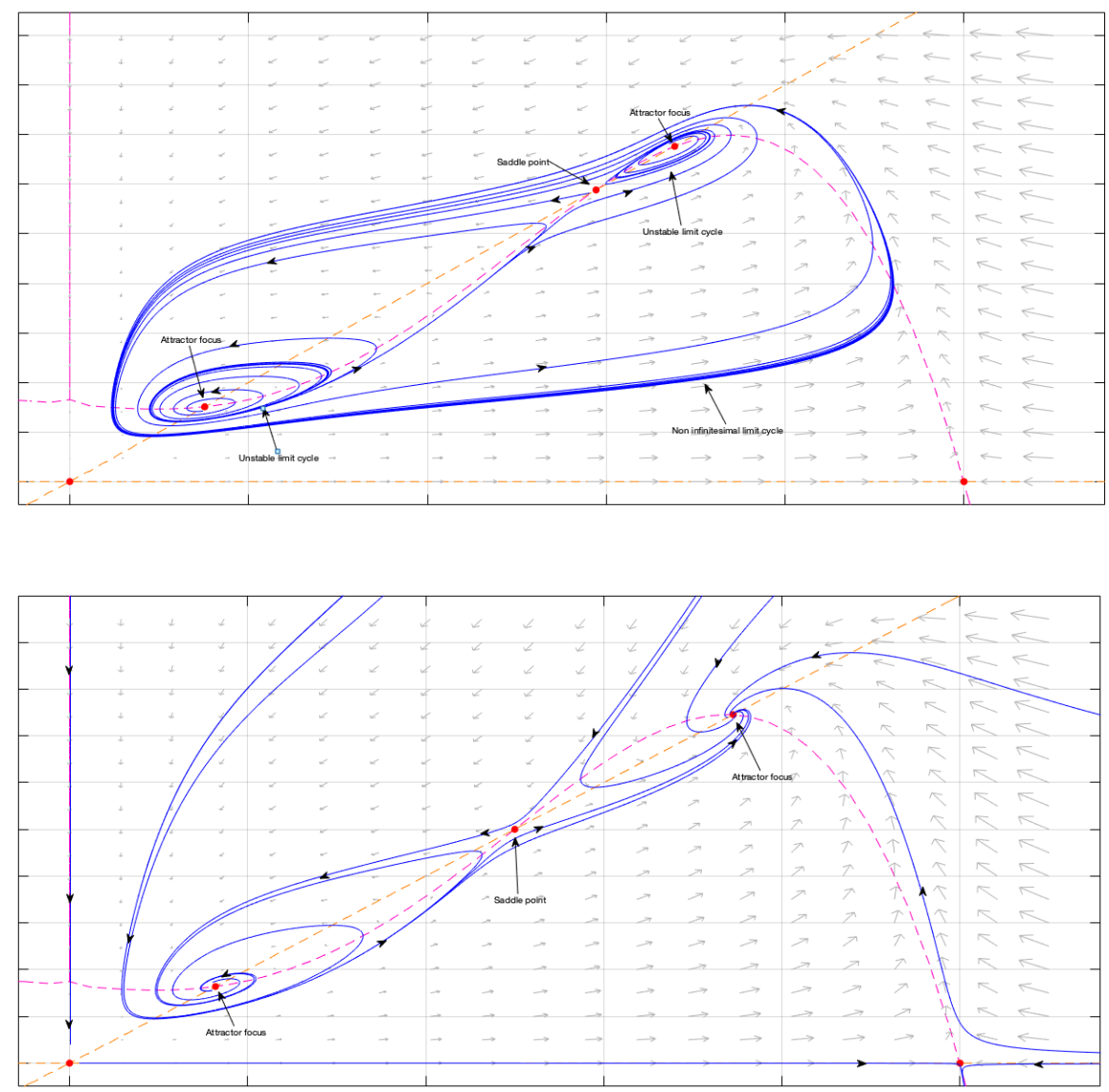